\title{
EMSL and Institute for Integrated Catalysis (IIC) Catalysis Workshop
}

November 17-18, 2010

Prepared for the U.S. Department of Energy's Office of Biological and Environmental Research under Contract DE-AC05-76RL01830

Pacific Northwest National Laboratory Richland, Washington 99352 


\title{
DISCLAIMER
}

This report was prepared as an account of work sponsored by an agency of the United States Government. Neither the United States Government nor any agency thereof, nor Battelle Memorial Institute, nor any of their employees, makes any warranty, express or implied, or assumes any legal liability or responsibility for the accuracy, completeness, or usefulness of any information, apparatus, product, or process disclosed, or represents that its use would not infringe privately owned rights. Reference herein to any specific commercial product, process, or service by trade name, trademark, manufacturer, or otherwise does not necessarily constitute or imply its endorsement, recommendation, or favoring by the United States Government or any agency thereof, or Battelle Memorial Institute. The views and opinions of authors expressed herein do not necessarily state or reflect those of the United States Government or any agency thereof.

\author{
PACIFIC NORTHWEST NATIONAL LABORATORY \\ operated by \\ BATTELLE \\ for the \\ UNITED STATES DEPARTMENT OF ENERGY \\ under Contract DE-AC05-76RL01830
}

Printed in the United States of America
Available to DOE and DOE contractors from the Office of Scientific and Technical Information,
P.O. Box 62, Oak Ridge, TN 37831-0062;
ph: (865) 576-8401
fax: $(865)$ 576-5728
email: reports@adonis.osti.gov

\begin{abstract}
Available to the public from the National Technical Information Service, U.S. Department of Commerce, 5285 Port Royal Rd., Springfield, VA 22161 ph: (800) 553-6847 fax: $(703) 605-6900$ email: orders@ntis.fedworld.gov online ordering: http://www.ntis.gov/ordering.htm
\end{abstract}

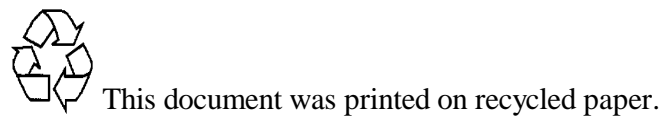


PNNL-20426

\section{EMSL and IIC Catalysis Workshop}

November 17-18, 2010

Prepared for the U.S. Department of Energy’s Office of Biological and Environmental Research under Contract DE-AC05-76RL01830

Pacific Northwest National Laboratory

Richland, Washington 99352 



\section{Executive Summary}

It is increasingly recognized that new research tools aided by advances in information and computation technologies are transforming the ways that science can be done. When appropriately combined with multidisciplinary research teams and research efforts focused on one or more important catalysis-related challenges, a framework for scientific discovery can emerge. The Environmental Molecular Sciences Laboratory (EMSL) located at Pacific Northwest National Laboratory (PNNL) is a national user facility that provides cutting-edge analysis tools and advanced computation, data processing, and storage and modeling capabilities. With a range of fundamental and applied research efforts, the Institute for Integrated Catalysis (previously the Institute for Interfacial Catalysis), or IIC, at PNNL facilitates collaborative research and development in catalysts for a secure energy future. Teams from EMSL and the IIC, working collectively with EMSL users, have the potential to accelerate scientific understanding of catalysis science with an appropriate eye on industrial application. Within the context of significantly accelerating scientific progress in research areas that address important societal problems, a workshop was held in November 2010 at EMSL to identify specific and topically important areas of research and capability needs in catalysis-related science.

Thirty-eight participants from 10 institutions, including eight international catalysis experts serving as an advisory panel, discussed research areas that would be high-impact and focused. They also identified research platforms needed to advance catalysis science and ways that EMSL and the IIC could partner to enable a new research paradigm with the potential to change the way that catalysis research is conducted. Recommendations were made for new instrumental and computational tools and high-priority research areas that would benefit from the synergistic combination of EMSL and the IIC. In particular, the need to expand EMSL's current instrument platforms to include additional in situ, real-time, high spatial resolution measurements was noted. In addition to building upon the intrinsic strength in oxide materials, additional research areas with a high national need were identified, including biomass conversion, $\mathrm{CO}_{2}$ conversion, and electrical-chemical energy transformation. The workshop was organized by Professor Charles T. Campbell, University of Washington; Charles Peden, IIC-PNNL; and Donald Baer, EMSL-PNNL. The names of workshop presenters, discussion leaders, and participants can be found in the appendices herein.

Workshop participants noted a joint program involving the IIC and EMSL with an appropriately chosen topical focus and suitably long enough duration would be well positioned to produce major scientific and technological impacts. Other important points addressed by participants included:

- EMSL has the ability to provide both expertise and tools for materials analysis under realistic catalytic conditions that could not be sustained at most universities.

- The ongoing maintenance and development of computer codes, such as NWChem, could provide as-yet unrealized robust state-of-the-art codes in a powerful way not possible at universities.

- EMSL has a unique set of platforms or tools to speed the link between theory and experiment.

- The IIC's considerable interaction with the industrial catalysis research and development (R\&D) community extends the potential range and speed of impact for important discoveries.

- The combination of PNNL, IIC, EMSL, and EMSL user expertise creates unique opportunities where coordinated and focused efforts could yield impactful results.

Although this type of focused team research may require some refinement or evolution in the way a user program is identified and supported at EMSL, it still resonated that EMSL, the IIC, and the approach to research within the national 
laboratory offered a solid basis for development and demonstration of a new research paradigm that would accelerate scientific and technological progress. 


\section{Acronyms and Abbreviations}

DOE

EMSL

IIC

NMR

PNNL

$\mathrm{R} \& \mathrm{D}$

STEM
Department of Energy

Environmental Molecular Sciences Laboratory

Institute for Integrated Catalysis (née the Institute for Interfacial Catalysis)

nuclear magnetic resonance

Pacific Northwest National Laboratory

research and development

scanning/transmission electron microscopy 


\section{Contents}

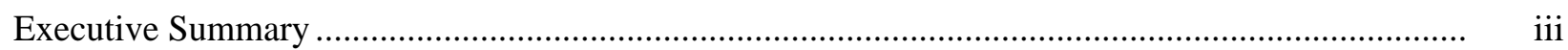

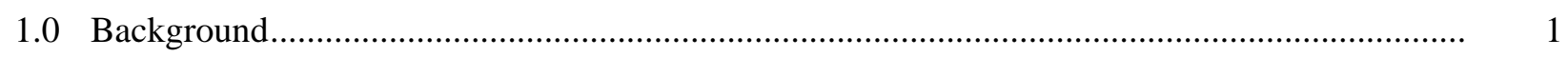

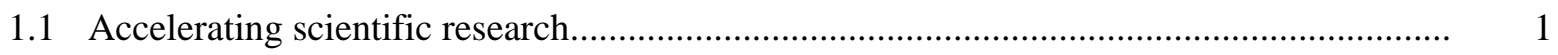

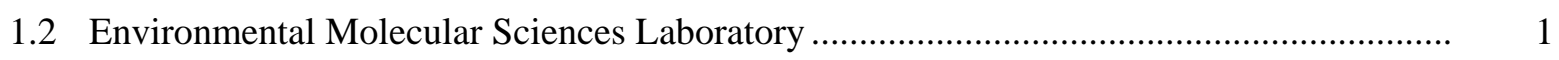

1.3 Institute for Integrated Catalysis ….............................................................................. 2

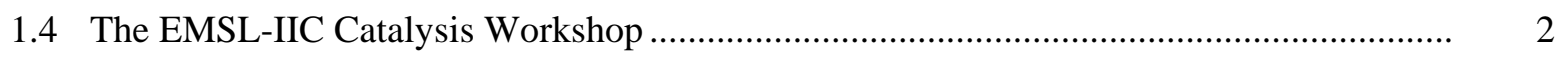

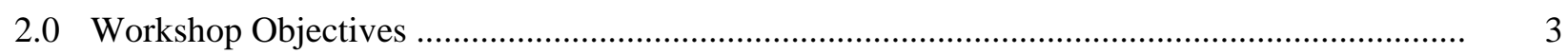

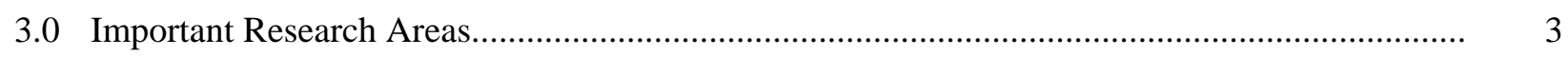

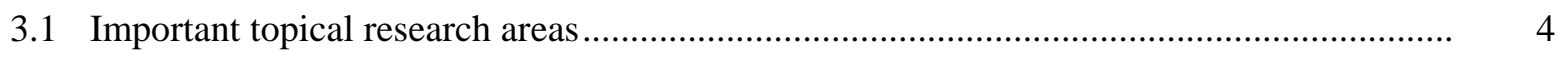

3.2 Important challenges to address ................................................................................ 4

3.3 Approaches/opportunities for advancing fundamental understanding .............................. 5

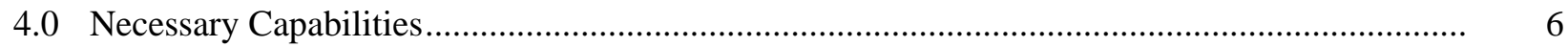

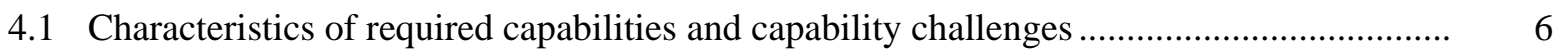

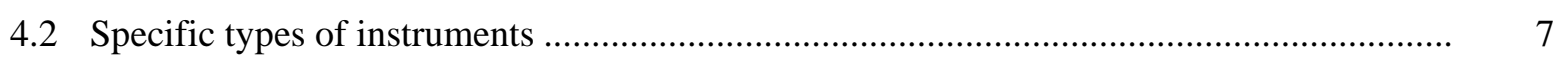

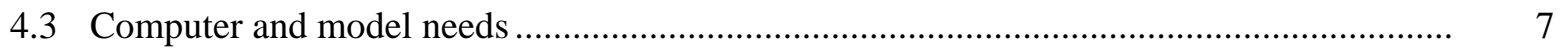

5.0 Roles for EMSL and IIC—Overall Recommendations …..................................................... 8

5.1 Capabilities for and focus on biomass conversions.................................................... 8

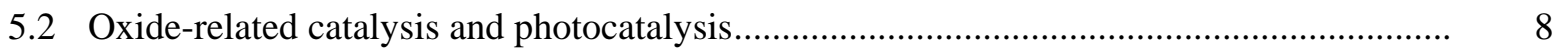

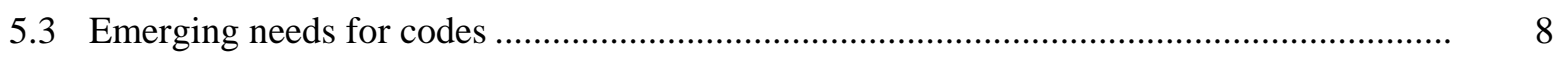

5.4 Roles for EMSL-IIC partnership and team research success …....................................... 9

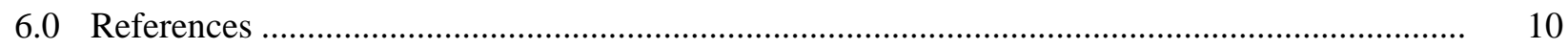

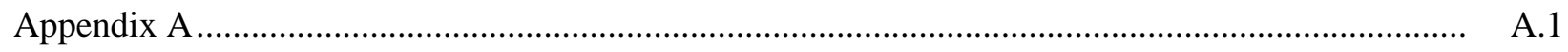

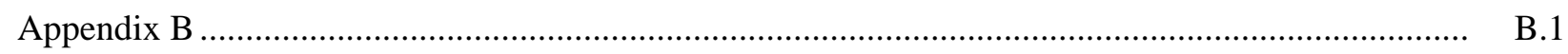

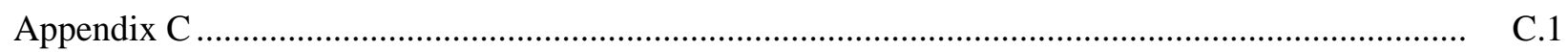




\subsection{Background}

\subsection{Accelerating scientific research}

Tackling the largest catalysis science and technology challenges requires new and efficient ways of performing research and development (R\&D), especially with respect to connecting the broader catalysis research community while recognizing the paradigm for funding research will likely change slowly, at best. The idea that research can or should be accelerated to address important societal problems is not new (Pielke and Byerly 1998), but it is garnering increased attention as the range of challenges that must be addressed grows (Whitesides and Deutch 2011). A variety of observers recognize that the way scientific research is executed keeps changing as the power of computers increases along with the concomitant surge in data generation (Science 2010, DOE 2008, and NSF 2004). The introduction to a summary of scientific research over the past decade in Science (2010) noted that:

In the past 10 years, new ways of gathering, analyzing, storing, and disseminating information have transformed science. Researchers generate more observations, more models, and more automated experimentation than ever before, creating a data saturated world. The Internet has changed how science is communicated and given nonscientists new opportunities to take part in research. Whole new fields, such as network science, are arising, and science itself is becoming more of a network-more collaborative, more multidisciplinary - as researchers recognize that it takes many minds and varied expertise to tackle complex questions about life, land, and the universe.

These advances in computation and high-speed networks along with the fantastic but often complex and sophisticated advanced experimental capabilities of modern science create important new opportunities and approaches for combining the expertise of different researchers and research teams to address intricate and challenging problems. An overall goal of this workshop was to examine how the Environmental Molecular Research Laboratory (EMSL) and the Institute for Integrated Catalysis (IIC) (until recently known as the Institute for Interfacial Catalysis) can facilitate collaborative research and the formation of teams of teams $\left(\right.$ teams $^{N}$ ) involving researchers from around the world to address major catalysis research challenges and to ascertain what tools and data infrastructure are needed to help make it happen.

\subsection{Environmental Molecular Sciences Laboratory}

EMSL is a national scientific user facility that provides integrated experimental and computational resources for discovery and technological innovation. EMSL's primary research focus is environmental molecular sciences to support the needs of the Department of Energy (DOE). However, the laboratory and its personnel also support research in the biological, chemical, physical, and computational sciences relevant to other governmental agencies and the nation.

EMSL opened its doors to users in 1997 and, for more than 14 years, has established a strong science foundation and demonstrated a robust and productive user program. Still, EMSL seeks to further focus research efforts to deliberately address national and global challenges related to energy and the environment. As a national user facility, EMSL is well positioned to focus research attention and efforts on important scientific challenges that address these major societal issues. EMSL's objective is to accelerate scientific innovation and discovery by transforming how scientific research is done.

Although much of the user-proposed research conducted in EMSL involves multiple instruments and technical capabilities, there are significant additional opportunities to advance science and speed scientific progress by appropriately designed experimental and computational research efforts, advanced data integration and sharing, and the 
formation of research teams ${ }^{N}$ focused on solving important problems. Furthermore, the EMSL user program can provide a process to assist in assembling teams with the diverse expertise to address important and challenging problems, especially those in catalysis research, because of the range and particularly relevant capabilities of EMSL resources.

\subsection{Institute for Integrated Catalysis}

Catalysis research and related tools were built into EMSL's foundation and have been one highly successful component of EMSL user research activities. As the quantity of catalysis research programs grew within EMSL and other parts of the national laboratory, Pacific Northwest National Laboratory (PNNL) initiated the Institute for Interfacial Catalysis (IIC) in 2005 with Professor Mike White as its first director. The IIC has become the largest non-industrial catalysis R\&D organization in the United States, with work spanning from basic to applied research. In May 2011, the IIC was renamed Institute for Integrated Catalysis, reflecting the merging of different subfields of catalysis science and engineering, with chemist Johannes Lercher, a professor at Technische Universität München in Germany, as its new director. The institute will maintain a stronger focus on the scientific challenges in securing a clean energy future. Through a broad and varied portfolio of DOE, other federal agency, and industry funding, IIC research provides critical new scientific understanding and practical catalyst material and process solutions for converting biomass to fuels and chemicals, new emission control technologies, conversion of chemical to electrical energy in fuels cells, and other energy and environmental areas. It oversees activities that involve a significant number of academic and industrial collaborators. Although highly successful, the IIC recognizes that being made up of a pragmatic collection of individual research projects in many areas sometimes limits its ability to apply the full range of expertise and capabilities available in the IIC, EMSL, and the broader catalysis R\&D community to attack important issues. Thus, the IIC, whose research approach involves the integration of fundamental and applied studies and theoretical and experimental sciences, has great interest in new, focused, and optimized methods to have an even larger impact on major catalysis-related problems. Three specific areas of research that would have significant impacts on energy production have been identified as important targets: 1) biomass conversion to fuels, 2) $\mathrm{CO}_{2}$ conversion to fuels, and 3) electrical-chemical energy interconversions. Each of these problems can be addressed, partly or fully, with the many and varied sets of tools available within the EMSL.

\subsection{The EMSL-IIC Catalysis Workshop}

To date, EMSL and the IIC have been resourceful and informal partners involving daily use of EMSL facilities and expertise for funded IIC research and, significantly, many joint efforts to enhance catalysis research capabilities within EMSL for the broader catalysis science community. As noted, the EMSL and IIC have mutual interests in finding new ways to focus efforts that speed scientific discovery and innovation to increase their respective impact on scientific problems of societal relevance. Because of their common interests, EMSL and the IIC anticipate forming a more formal partnership to develop capabilities and research approaches that enhance scientific efforts aimed at advancing the ability to control chemical transformations and electrical-chemical energy interconversions. It is the contention of leaders in both EMSL and the IIC that a coordinated effort linking multiple state-of-the-art instruments with computational simulations, coupled with new technology for collaborations between scientists at various locations, can lead to rapid scientific and technological progress. Such significant advancements in both experimental and theoretical approaches are consistent with recommendations from recent DOE- and National Science Foundation (NSF)-sponsored workshops (DOE 2008 and NSF 2004). The EMSL-IIC Catalysis Workshop described in this report was held as part of the process of moving from the important general recommendations from previous workshops toward identification of more specific science topics and research capabilities that can be combined in an organized effort that advances the ability to design catalysts for specific purposes in energy production, storage, and conversion. 


\subsection{Workshop Objectives}

Academic, national laboratory, and industrial research groups of various types have highly successful research teams and projects advancing both fundamental understanding and developing next-generation catalysts and catalytic processes for industrial use. This workshop's overall objective was to identify specific areas where, with access to appropriate capabilities, an assembly of multi-institutional teams with diverse expertise working on a common project or focus area would be able to address an important catalysis problem or challenge in a way that could significantly accelerate progress.

The participants in the workshop were asked to answer three questions:

1. What are the "next-generation" capabilities that we should be considering as future EMSL user capabilities to help advance catalysis and catalysis science?

2. Are there one or more specific fundamental catalysis research areas where EMSL teaming with the IIC and a variety of users could focus efforts to encourage scientific advancement, particularly in areas relevant to energy production, storage, and/or conversion? Such efforts might include research campaigns or some other form of focused effort.

3. What are the best mechanisms to use EMSL resources in this arena to maximize impact and speed progress?

The workshop included short presentations on the current instrumentation and technologies available in EMSL, as well as some projections of what advances might be possible in the future, an overview of some of the current science conducted in EMSL and at the IIC, a short review of high-priority areas for catalyst research identified by the DOE's Office of Science, and breakout sessions where participants were separated into four discussion groups focused on the aforementioned three workshop questions.

Workshop participants included EMSL staff members, PNNL staff members with catalysis expertise and interests, and an external advisory team composed of internationally recognized catalysis researchers. In addition to participating in the discussion, the external advisors assembled their initial thoughts on important topics and critical capabilities prior to the meeting.

\subsection{Important Research Areas}

The workshop began with a succinct overview, provided by Professor Charles T. Campbell, of the most recent DOE Basic Energy Science (BES) workshop on catalysis. After this introduction, advisory participants in the workshop were provided the opportunity to present their initial thoughts on important areas. The various perspectives introduced a diversity of viewpoints that made each individual's input highly valuable to the global context. The workshop also broke into four discussion groups, with each presenting a summary of the topics and issues deemed important for advancing catalysis science and addressing important practical problems. These recommendations constitute three categories: 1) important topical areas ripe for impactful research; 2) fundamental challenges that, if successfully addressed, would significantly advance catalysis science and the design of next-generation catalytic materials and processes; and 3) nextgeneration experimental and computational approaches that will be necessary to address these fundamental science challenges. 


\subsection{Important topical research areas}

Catalysts play major roles in many processes important to chemicals and fuels production, energy conversion and storage, and environmental protection. Designing catalysts that improve the efficiency of chemical processes, remain active for long periods of time without fouling, and optimize outcomes for specific reactions can have a major impact on challenges related to energy production, use, and storage. During the workshop, three important topical areas were identified where new or advanced catalysts would have high impact.

Biomass Conversion-The conversion of biomass to chemical feedstocks has the potential to decrease reliance on petroleum products used during production of various chemicals important for modern society and in providing renewable fuel sources. The challenges associated with biomass conversion include the need to reduce costs and improve processes for conversion of a variety of biomass types - many of which are currently intractable or highly expensive. The design of robust and less expensive catalysts to assist this process would be a critical component for advances in biomass conversion. A particular challenge for catalysts associated with biomass conversion is stability and efficiency in an especially complex chemical environment that often involves high concentrations of water because water is known to cause major stability issues for most known catalysts during use.

$\mathrm{CO}_{2}$ Conversion-The amount of $\mathrm{CO}_{2}$ released by combustion of petroleum products remains a major concern and, regardless of source, is associated with global climate change. The ability to convert $\mathrm{CO}_{2}$ to a fuel efficiently would be a major step toward establishing long-term sustainable energy systems. Because energy is required for such a conversion, a photoactive catalyst might be a promising choice, with the energy needed to drive the desired reactions provided directly by the sun.

Electrical-Chemical Energy Conversion-By incorporating various energy production systems that do not produce energy on demand (e.g., wind and solar), the need for energy storage increases. Storing energy in chemical bonds is one of the most efficient methods of addressing this critical issue. Thus, efficient systems to convert electrical energy to chemical energy are indispensable. Catalytic materials and processes are essential for electrical-to-chemical energy interconversion systems, including fuel cells and batteries.

\subsection{Important challenges to address}

Success in the described research areas requires significant advances in the ability to design, optimize, and produce the necessary new generation of catalysts and catalytic processes. Although some challenges may be specific to one particular type of catalyst, there are also some general challenges that, if successfully addressed, would enable advances in the design and use of several types of catalysts. Several of these important challenges were identified at the workshop and include:

New approaches to catalysis design and testing - Over the next decade, research for catalysis science will develop with an increasing integration of experiment, theory, catalyst design, characterization, and testing. Combining the expertise within EMSL and the IIC, there is the ability to speed progress by "short circuiting" the interaction between theory and experiment and having the appropriate characterization and testing capabilities available. This approach includes having a full range of capabilities and tools to identify and evaluate the importance of the elementary steps of catalytic reactions. A significant objective is to understand structure/function relationships for catalytic processes and incorporate them into the design of next-generation systems.

Understanding the structure and properties of complex oxides-As both supports and active catalysts, oxides are important in many catalytic systems and are an area of research strength within EMSL and the IIC. Oxides of increasing 
complexity and sophistication are being examined, and knowledge of the phase separation/segregation and phase transformation processes in these complex oxides will be important for understanding and predicting their properties. It was pointed out that catalysts often operate on the "edge," where material stability at different temperatures and within different environments may determine catalyst performance or lifetime. For example, in the area of energy conversion, phase transformations under different voltages impact properties such as battery lifetime and ease of recharging.

Determine fundamental reaction mechanisms with atom, energy, and time resolution-Reactions kinetics are fundamental in understanding catalytic behaviors. Knowledge about sites of reactions, the temperature dependence of the process, and time dependence of reactions is essential to understanding and controlling catalyst behaviors. Models must be developed that can rationalize kinetics in working catalytic systems. Part of comprehending reaction kinetics requires understanding the complex in situ dynamics of the materials in working environments. Addressing this challenge will require advancing experimental and theoretical capabilities and improved theory-modeling-experimental coupling.

Homogeneous catalysis and homogenous catalysts linked to surfaces-Biomass conversion involves highly complex conversion pathways and reactions in liquids. However, the introduction of homogeneous and/or heterogeneous catalysts may overcome some of the process difficulties. Specifically, the ability to design homogeneous catalysts and attach them to specific surfaces may provide spatial stability and possibly introduce multifunctionality.

Design of multifunctional catalysts - Catalytically enhanced processes often involve more than one type of catalyst and may depend on materials or materials systems that enable a variety of functions. Applications for which multifunction is important include photocatalysis, solar-thermal systems catalysis, fuel cell electrodes, and chemical sensors. For these and other types of systems, the functionality required may include catalytic activity, specific electrical properties (conductivity, insulating, or semiconducting), specific ionic conductivity (e.g., $\mathrm{H}^{+}, \mathrm{O}^{2-}$ ), optimized band gap for visible light adsorption, high thermal stability, ferroelectricity, or ferromagnetism.

\subsection{Approaches/opportunities for advancing fundamental understanding}

In addition to addressing important topics and identified challenges, significant advances in understanding may be achieved by applying unique approaches in conducting catalysis research. Along with the topics already addressed, the workshop participants noted two opportunities for approaches toward advancing catalysis research, which seemed particularly well matched to a joint EMSL-IIC effort based on the expertise available and the promise of high-impact results (as follows).

Combining studies of single crystal surfaces and single facet nanomaterials-The ability to form high-surface-area nanomaterials (presenting a single surface structure) creates new opportunities to study catalytic reactions on specific surfaces. By combining high-surface-area, single-facet material studies with single-crystal surface studies, it should be possible to conduct both traditional surface studies and include measurements, such as nuclear magnetic resonance (NMR) and optical spectroscopy, which require high surface area. Single-facet nanomaterials also will enable new types of detailed studies to be conducted in solution. They combine the advantages of site homogeneity offered by single crystals (leading to fewer, sharper, more intense, and easier-to-interpret peaks in any measurement) and high total surface area offered by powders (which leads to better signal-to-noise ratio in any measurement).

Building up the complexity axis_-Baseline computational and experimental studies of well-defined model materials can be used to determine simple structure-function relationships and build predictive models. Upon this platform, increasingly complex materials can be studied, and parameters can be added to the predictive model, generating a stepwise knowledge base in an effort to delineate the factors influencing the most highly complex heterogeneous systems. 
This approach will allow specific kinetic, spectroscopic, and structural features to be elucidated as a function of the whole rather than a simple linear combination of single components.

\subsection{Necessary Capabilities}

A diversity of tools that could be used to significantly advance catalysis science was discussed. The previously addressed topics remained foremost in the workshop participants' minds. Several overarching domains provide a context for these recommendations. Due to the intrinsically complex conditions under which catalysis takes place, it would be advantageous to perform measurements under similarly complex conditions in solution and gaseous environments. Although there is no single "ideal" solution for this requisite approach, there are a variety of intermediate steps that can and are being taken to obtain improved information for such conditions. It is widely recognized that catalysis is inherently a dynamic process for which the kinetics of reactions have both economic importance and provide the basis for understanding the chemical reactions. Many of these general challenges were broached. In addition, workshop participants categorized specific instruments, instruments types, or instrument combinations as highly important and useful, including: development of in situ transmission electron microscopy (TEM) capabilities; constant-flow, hightemperature magic angle spinning (MAS) NMR; and additional advances in the broad areas of surface characterization and reactivity. Workshop participants were encouraged to complete the ongoing activities and push forward, as outlined in the recommendations summarized as follows:

\subsection{Characteristics of required capabilities and capability challenges}

- Realistic environments

- Ability to make measurements in liquid-state environments (particularly important for biomass conversion)

- Novel in situ tools

- In situ following of oxidation/reduction during catalytic processes

- Examining phase changes as a function of temperature, time, environmental conditions, and electric potential.

- Dealing with complex systems

- Tools to study multi-phase and multifunctional catalysts

- Reactivity mapping of complex heterogeneous oxide surfaces

- Improved tools for studying complex liquid environments.

- Time dependence and dynamic measurements

- Increased ability to make dynamic measurements and to measure kinetics, including increased speed of data acquisition and analysis

- Measurements across multiple time scales, especially focused on approaching increasingly faster time scales.

- Capability-enhanced approaches

- Increased coupling of theory and experiment

- Need model-guided synthesis of new catalysts

- Must have a "method or center" to demonstrate new capabilities to the community 
- $\quad$ Need higher resolution for analysis of small objects and specific reaction sites; higher sensitivity for lower concentrations; and faster data generation, collection, and analysis

- One important need for high spatial resolution involves establishing property distributions based on the behavior of individual objects rather than an average of the total ensemble. Sometimes, the interesting and important properties are the outliers, i.e., those significantly far from the average.

\subsection{Specific types of instruments}

- Specific instruments or needed advances in instrumental capabilities were recommended in several areas.

- Advances in electron microscopy, fast measurements, and analysis

- Advanced low-energy ion scattering (LEIS) for true outer surface measurements

- Need to extend the information about surfaces, particularly under realistic conditions

- High spatial resolution (tip enhanced) scanning infrared spectroscopy

- High-pressure and spin-sensitive scanning/transmission electron microscopy (STEM)—particular role for EMSL and a user facility in this area

- Environmental chamber for the nanospray Desorption ElectroSpray Ionization (nanoDESI) and other non-vacuum mass spectrometry methods to be applied to catalysis studies.

- Combinations of instruments

- Combine STEM and calorimetry

- Combine atomic force microscopy (AFM) and NMR

- Fast-switching kinetics for biomass conversion and calorimetry

- Combinations of surface-sensitive and other related tools that work in operating environments.

\subsection{Computer and model needs}

There were several needs identified in the theory, computation, and modeling areas, but major questions arose around the ability to link the current code types used in the catalysis community to the more advanced molecular codes commonly incorporated into NWChem. Although NWChem has much of the functionality currently used and desired by the catalysis community, NWChem is virtually unknown and unused by much of the catalysis community. NWChem contains a plane wave code that is capable of treating surfaces and interfaces on par with other plane wave codes, such as VASP or GPAW, and includes the functionality typically used by the simulation community for investigating catalytic systems. Included are methods for finding saddle points using a quasi-Newton method, reaction pathways using the Nudge Elastic Band (NEB) method, and potential of mean forces (PMFs) using constrained ab initio molecular dynamics and metadynamics. Currently, Vanden-Eijnden's string method is being implemented. NWChem also supports python scripting methods that allow easy implementation of new functionality developed by other groups such as the Atomic Simulation Environment (developers of DACAPO and GPAW) or Phonopy. Recently, it was demonstrated that the plane wave part can scale from tens of processors to tens of thousands of processors, which shows it can be used for a variety of system sizes, ranging from small to large jobs. Regardless of the presence of these codes in NWChem, these capabilities and functionalities are clearly unknown to the broader heterogeneous catalysis community, and there is a clear need to address the following: 
- Significantly increase the visibility of NWChem in the catalysis community and demonstrate the full range of codes and functionality available that are relevant to that community

- Facilitate the linking of lower cost methods to some of the higher-level models also available in NWChem

- Highlight how to perform low-cost screening calculations to identify where more accurate calculations may have highest value

- Work with the catalysis community to identify the advanced code developments that can be included in NWChem, which can advance the modeling tools available for catalysis research.

\subsection{Roles for EMSL and IIC-Overall Recommendations}

Three general recommendations were voiced repeatedly during many of the discussions regarding how the EMSL-IIC partnership should focus attention in the effort to advance catalysis science.

\subsection{Capabilities for and focus on biomass conversions}

Biomass conversion and alternative fuel production was highly recommended as a complex and timely topic worthy of intense focus. It also was recognized that although many existing EMSL capabilities would be useful in this area, there would be additional need for new capabilities development. This area includes several interesting challenges that unite aspects of both interfacial and biological science to deal with an extremely important and difficult topic.

\subsection{Oxide-related catalysis and photocatalysis}

Workshop participants noted that oxide surface chemistry is an internationally recognized strength for EMSL and the IIC. In addition to catalysis, oxides are a highly versatile class of materials with important implications in many areas. The research strengths related to oxides provide a foundation for advancing many areas of catalysis, upon which additional work should be built. An expanded focus on photocatalysis, an important multifunctional material (chemical reactions and light harvesting), is a logical extension of current research.

\subsection{Emerging needs for codes}

NWChem and the EMSL Chinook supercomputer can be highly valuable resources for the catalysis community. However, as noted, NWChem is not well established in the catalysis community, and specific efforts to remedy this are needed. The workshop participants indicated that an apparent emphasis of NWChem on the highest end users running advanced molecular codes has limited the appeal of NWChem to the catalysis community. In addition to making the codes known in the catalysis community, EMSL needs to work with the catalysis community in identifying the nextgeneration codes that can help advance catalysis research. 


\subsection{Roles for EMSL-IIC partnership and team research success}

In several different discussions, workshop participants indicated there were multiple ways that EMSL (as a sciencefocused user facility) and the IIC (as a research center that couples fundamental and applied research) provided important attributes that could be optimized to enable significant advances in catalysis science. These characteristics include:

- Access to multi-disciplinary teams of varied catalysis, materials science, and instrumentation expertise

- The ability to provide long-term continuity to the research direction

- Access to world-quality experts operating state-of-the-art instrumentation

- Ability to appropriately maintain and expand computer codes that operate on powerful systems

- The capability to maintain one-of-a-kind instruments operating under extreme or in situ conditions that cannot be replicated at universities

- Power to establish working groups coalesced around domains of high need-computational, experimental, and/or defined problems transecting multiple disciplines

- Ability to maintain an integrated tool set

- Ability to store and share data that enable a new paradigm of catalysis research.

Other specific focus items include:

EMSL-IIC-Academic-Industry links-Because of the importance of catalysis to industry and the knowledge base contained within industry, long-term success and impact will require industry partnerships. Similarly, many excellent centers of academic research can provide essential fundamental insights for solving critical catalysis problems.

Furthermore, such connections involve the next generation of catalysis scientists and engineers who are critical for the future of catalyst science and technology. For example, participation in catalysis research with wide ranging, advanced EMSL capabilities provides highly important educational opportunities.

EMSL and IIC need to guide/encourage collaborative team formation and show the impacts of doing science in a new way - The formation of productive "teams of teams" is not easily accomplished. It will require considerable effort and encouragement to make this approach work. The workshop advisors thought it was especially appropriate and, possibly, an essential approach as well as an important role that EMSL as a user facility can lead. However, there are a variety of technical, sociological, and communication barriers that will need to be overcome. The combination of the IIC, EMSL, and the national laboratory environment can be used demonstrate how science can be done differently.

EMSL needs to develop a long-term collaborative proposal process-The normal mode of relatively short-term user proposals is not a model that will allow for significant advances in areas of high complexity. EMSL needs to develop a mechanism that allows longer-term collaboration in specific target areas. This approach includes the requirement that new partners or team members be plugged into the problem as the science or technology need dictates.

Data sharing is vital-The ability to share data at all levels and various stages of research is essential to accelerating the rate of research progress. The new MyEMSL platform can provide some basis for this, but it likely will require expansion and development of data protocols.

Existing codes and improved codes that link better to the catalysis community—Some code development, demonstrated deployment, and energetic efforts to help establish and promote NWChem to the catalysis community are needed. 


\subsection{References}

DOE - Department of Energy. 2008. "Basic Research Needs: Catalysis for Energy.” U.S. Department of Energy Basic Energy Sciences Workshop, August 6-8, 2007, Bethesda, Maryland. Accessed March 25, 2011 at http://www.science.doe.gov/bes/reports/files/CAT_rpt.pdf.

NSF - National Science Foundation. 2004. "Future Directions in Catalysis: Structures that Function at the Nanoscale.” National Science Foundation Workshop on Future Directions in Catalysis: Structures that Function at the Nanoscale, June 19-20, 2003, Arlington, Virginia. Accessed March 25, 2011 at http://www.che.caltech.edu/nsfcatworkshop/\#Reports.

Pielke Jr RA and R Byerly Jr. 1998. “Beyond Basic and Applied.” Physics Today 51(2):42-46.

Science. December 17, 2010. “Stepping Away From the Trees For a Look at the Forest.” 330(6011):1612-1613.

Whitesides GM and J Deutch. 2011. “Let’s get practical.” Nature 469(7328):21-22. 


\section{Appendix A}





\title{
Appendix A: Agenda
}

\author{
EMSL and IIC \\ Catalysis Mini-Workshop \\ November 17 and 18, 2010
}

November 17

6:00 pm Anthony's-Vineyard Room, Kickoff Dinner at 6:30 pm setting the stage

- A vision of the EMSL Future

- Set agenda and meeting objectives

Three questions:

i) What is/are the "next" generation capability/s that we should be considering in EMSL to help advance catalysis and catalysis science?

ii) Are there one or more particular areas where EMSL can and should focus efforts to encourage scientific advancement, particularly as relevant to energy production or storage? Such effort might include research campaigns (and whatever that can mean) or some other type of focused effort (the EMSL 2.0 ideas).

iii) What are the best mechanisms to use EMSL resources in this arena to maximize impact?

\section{November 18}

\section{7:30 am Badging (foreign nationals ETB, US citizens EMSL Lobby)}

8:00 am l) Welcome and plans for the day - EMSL Boardroom

- EMSL Future Vision - Allison Campbell

8:20 am $\quad$ II) Current status and directions - EMSL Boardroom

- Catalysis as part of the Science of Interfacial Phenomena Science Theme (Don Baer)

- The vision of the Institute for Interfacial Catalysis (Chuck Peden)

- IIC/EMSL Partnership Possibilities (Chuck Peden and Don Baer)

- Short summary of (2007) BES-sponsored catalysis workshop US catalysis research community's 'grand challenges' (Chuck Peden or Charlie Campbell)

- Workshop participants thoughts on potential 'hot' directions in the next 10 years

- Thoughts on important research directions in catalysis and an EMSL role (Charlie Campbell)

- Table discussion and idea collection on important areas and needs

11:30 am III) Instrumental Capabilities in EMSL - EMSL Boardroom

- Overview of New Capabilities (Don Baer)

- NMR recent developments and future directions (Karl Mueller)

- Evolving capabilities in microscopy and potential advancements (Bernd Kabius)

12:20 pm III) Instrumental Capabilities in EMSL (continued)

- Increasing the experiment theory link - Direction for NWChem (Eric Bylaska)

- Surface Science (Theva)

- Optical Spectroscopy (Chuck Peden)

- Workshop participants ideas of capabilities (beyond those discussed) they would like to see at such a user facility

- Table discussion of potential gaps, important opportunities, barriers to progress, and ripe targets 
2:30 pm IV) Identifying Research Priority Research Targets - EMSL Boardroom

- Initial research campaigns as an example of an interaction mechanism (Don Baer)

- Table brain storming session of high priority areas are appropriate for EMSL related research team activities

3:00 pm V) Pulling things together - summary and comments - EMSL Boardroom

3:30 pm Non-PNNL Participant Discussion of EMSL Use Model - EMSL Boardroom

Charlie Campbell Discussion Leader - (EMSL user options. Is there better way to evolve new instrumentation capabilities so they have greater impact? Are there formal partnerships that should be established?)

4:30 pm End 


\section{Appendix B}





\section{Appendix B: Meeting Participants}

Advisory Panel

Charles T. Campbell (Co-Chair), University of Washington

Abhaya Datye,

University of New Mexico

Graeme Henkelman,

University of Texas

Raul Lobo,

University of Delaware

William Schneider,

University of Notre Dame

Len Spicer,

Duke University

WT Eddy Tysoe,

University of Wisconsin

John Vohs,

University of Pennsylvania

EMSL

Don Baer (Co-Chair)

David Hoyt

Theva Thevuthasan

Karl Mueller

Chongmin Wang
Nancy Washton

Igor Lyubinetsky

Ray Teller

Amity Anderson

Niri Govind

Karol Kowalski

Bernd Kabius

Hongfei Wang

Allison Campbell

Bill Shelton

Eric Bylaska

PNNL

Chuck Peden (Co-Chair)

Yong Wang

David King

Jun Lin

Michael Henderson

Roger Rousseau

Janos Szanyi

Zdenek Dohnalek

Dongha Mei

Bruce Garrett

Doug Ray

Jean Futrell

Julia Laskin

Dan Dubois

Meeting Support and Administration

Laura Kuprat

Charity Plata 



\section{Appendix C}





\section{Appendix C: Sample Invitation Letter}

Dear Participant,

The Environmental Molecular Sciences Laboratory (EMSL) DOE User Facility and the Institute for Interfacial Catalysis (IIC) at PNNL are forming a partnership to develop capabilities and approaches that enhance scientific efforts directed toward advancing our ability to control chemical transformations and chemical-electrical energy inter-conversions.

Consistent with recommendations from recent DOE- and NSF-sponsored workshops, we contend that a coordinated effort linking multiple state-of-the-art instruments with computational simulations can lead to rapid scientific and technological progress, and that significant advancements in both experimental and theoretical approaches are still required. To make progress on these challenges, we would like to invite you to participate in a full day workshop to be held in EMSL on November 18, 2010.

The specific objectives of the workshop are to address three topics:

- What is/are the "next" generation capability/s that we should be considering as future EMSL user capabilities to help advance catalysis and catalysis science?

- Are there one or more specific fundamental catalysis research areas where EMSL can and should focus efforts to encourage scientific advancement, particularly as relevant to energy production, storage, or conversion? Such efforts might include research campaigns or some other type of focused effort.

- What are the best mechanisms to use EMSL resources in this arena to maximize impact?

The workshop will involve short presentations on the current instrumentation and technologies available in EMSL (as well as some projections of what might be possible in the future), an overview of some of the current science conducted in EMSL and at the IIC, a short review of high priority areas for catalytic research identified by the DOE Office of Science, and break-out session discussions focused on the above three workshop objectives. A short written summary of the workshop recommendations will be prepared.

As an invited workshop participant we would cover your reasonable travel expenses for the meeting. The workshop will be preceded by an orientation dinner the evening of November 17. You would be able to depart Richland after about 6 pm on Nov 18 or the morning of Nov 19.

Please confirm your ability to participate in this workshop by sending an email to Laura Kuprat (laura.kuprat@pnl.gov).

Sincerely,

Donald R. Baer

EMSL Lead Scientist

for Interfacial Chemistry

Interim EMSL CSO
Charles H. F. Peden

Laboratory Fellow

Interim Director of the IIC

PNNL
Charles T. Campbell

Lloyd E. and Florence M. West

Professor of Chemistry

University of Washington 